\title{
Towards an Open Authoring Tool for Accessible Slide Presentations
}

\author{
Mirette Elias ${ }^{1}$, Abi James ${ }^{2}$, Steffen Lohmann ${ }^{3}$, Sören Auer ${ }^{4}$, and Mike Wald ${ }^{2}$ \\ 1 University of Bonn, Bonn, Germany \\ melias@uni-bonn.de \\ 2 University of Southampton, Southampton, UK \\ a.james@soton.ac.uk, mw@ecs.soton.ac.uk \\ 3 Fraunhofer IAIS, Sankt Augustin, Germany \\ steffen.lohmann@iais.fraunhofer.de \\ 4 Technische Informationsbibliothek, Hannover, Germany \\ soeren.auer@tib.eu
}

\begin{abstract}
Creating and sourcing accessible Open Educational Resources is a challenge. Although slides are one of the primary forms of educational resources, there has been little focus on what is required to make slides containing different media accessible and how to encourage authors to improve accessibility. This paper examines the components within slide presentations that impact accessibility and will evaluates six different approaches for encouraging authors to add accessibility issues. Authors indicated a preference for being encouraged and guided to resolve issues rather than allowing for automatic corrections.
\end{abstract}

Keywords: Open Educational Resources · OpenCourseWare · Slide Presentations · Accessibility · Inclusive Design · Slide Authoring · SlideWiki.

\section{Introduction}

Open Educational Resources (OERs) are openly licensed and freely available learning materials that can be used in e-learning contexts and beyond. One of the key advantages of OERs is that the license allows for the content to be reused, remixed, and repurposed [8]. Often, OERs are published on the Web in the form of OpenCourseWare (OCW), with resources organized into courses and complemented by tools for collaboration and evaluation. Learners may include those with disabilities who encounter barriers to their accessibility needs and preferences. These needs and preferences should be addressed by OCW systems that aim to be inclusive and accessible to all.

To ensure OERs are accessible, it is important to provide teachers and learners with disabilities with appropriate user interfaces (UIs) for reading, browsing, and authoring the materials. An OER can be represented in various formats, including text documents, slides, videos, and audio files. While considerable research has been conducted on the accessibility of individual media and text documents [11], one of the most common e-learning formats is slides for use in lectures and other teaching contexts. 
Slides are created to represent information concisely with a structured layout in order to help learners easily recall knowledge. A slide deck comprises of a collection of slides which can make up a course, lecture, or any other form of presentation on a specific topic. One slide can be considered to be the equivalent to a paragraph of text in that it should convey a single topic or concept. However, whereas a paragraph usually only contains text, a slide can include different types of media, such as images, tables, audio, and videos. Slides are also widely used independently of a presenter, as they provide a readable and printable version of the content. Furthermore, they can be exported to other types of formats, such as static PDF documents or video slideshows.

Slides can pose particular accessibility challenges due to the frequent use of images, bulleted lists, and tables. In addition, the spatial layout of content on a slide is often used to convey information. The experience for a disabled user accessing these different types of content can be highly dependent on the quality of the accessibility information (such as the captions, audio descriptions, and 'alternative' text) used to annotate the slide. While some annotations can be automated, the authors of the slides are usually most suited to undertake this task, as they are the subject experts.

This work addresses the following research question: What accessibility annotations should be included in a presentation slide and how can authors be encouraged to undertake actions to improve the accessibility of their slide content?

This research is conducted in the context of the SlideWiki EU project $^{5}$, which involves the development of a large-scale accessible OCW platform for OERs. The platform provides a means for creating and presenting slides online, in HTML, where authors can collaboratively edit and share their content. This offers the opportunity for the OERs to be accessible to assistive technologies, which can be used by authors and learners with disabilities. It is therefore necessary that both the content and the platform conform with digital accessibility standards. In addition, as the platform provides a collaborative slide creation tool, the accessibility of the content must be maintained even when authors edit and create different versions of the slides. Throughout the project, feedback on the platform design and use has been provided by a range of organizations involved in trials. Our methodology is organized into four steps:

1. Define the main components of the slides, and the accessibility needs for each component, to enable the content to meet the relevant accessibility guidelines ([4], [5], [7], [9]) and IMS Access For All (AfA) specification [2].

2. Identify which of these accessibility needs can be met through functionality within the slide creation tool and which will require action by content authors.

3. Identify approaches that can be used within a slide creation tool for ensuring authors undertake the necessary actions to ensure their content is accessible.

4. Gather feedback from authors on prototypes of the approaches that could be developed to improve the accessibility of their slides.

\footnotetext{
${ }^{5}$ https://slidewiki.eu
} 


\section{Accessibility Requirements of Slides}

As a first step to identify the accessibility requirements of slides, an analysis was undertaken of the relevant components and properties of slides and decks. A deck has three components: 1) meta-data, 2) outline, and 3) slides.

The deck meta-data defines the properties of a deck (e.g., language, date, topic). It also contains the theme defining the visual presentation of the content within the deck. Each theme uses Cascading Style Sheets (CSS) to set the font size, font type, font color, and background color. As the theme is used to set the visual elements of a deck, this can be used to manage accessibility by offering color and font combinations that meet accessibility guidelines. The default theme of the platform includes high contrast and common color combinations for those visual difficulties. The inclusion of additional meta-data concerning the accessibility of a deck and its content may also be useful for educators when searching for OERs. Such meta-data could be used to report decks that contain slides that have been checked for accessibility or to report the complexity of the text within the slides. This could be linked to the needs for learners to filter search results [6].

The outline refers to the structure and organization of the slides within the deck, which is important for users to be able to navigate through the slides and recognize the structure of the presentation using different assistive tools. Each slide in the index contains an ID and a name, which is human-readable when viewing the structure of the deck. The slide name is equivalent to a page or document title and is independent of the title used within the slide content. To meet accessibility guidelines, authors should be encouraged to give each slide within a deck a unique name.

A slide is the fundamental part of a deck; it can be composed of:

- The slide layout, which defines the location of the different content components within the slide and may also convey meaning.

- The slide content, which is made up of elements that may contain a heading, normal text (a short paragraph), a list, symbols and equations, tables, charts and images, hyperlinks, or embedded media such as a video.

A slide can only be considered to be fully accessible when each of the content elements within it meets the accessibility requirements for that type of media. In addition, the information indicated by the layout must be conveyed appropriately through the reading order and accessibility annotations. Predefined layouts, for example, with inputs boxes using predefined styles for the slide heading and a box for text and list content, can assist authors with managing the reading order if they are encouraged to use them. However, there is always a risk that authors will convey information through the layout (for example, if they use a number of components to create a diagram) and this will need to be described to readers who rely on non-visual access through accessibility annotations.

In order for the content created within the slide editor to be accessible, the following must be met: i) comply with accessibility guidelines, and ii) allow authors to annotate the content with additional accessibility information [1]. 
The first step taken to meet these requirements was to select an authoring toolbar that conforms to the W3C Authoring Tool Accessibility Guidelines 2.0 (ATAG 2.0) [3]. While such an authoring tool can generate accessible content, a review of the potential slide content elements identified six areas that would require input from the slide author, as outlined in Table 1.

Table 1. Accessibility requirements for the slide content elements

\begin{tabular}{|c|c|c|}
\hline $\begin{array}{l}\text { Slide content } \\
\text { element }\end{array}$ & $\begin{array}{l}\text { Accessibility added by the } \\
\text { system }\end{array}$ & $\begin{array}{l}\text { Author actions required to } \\
\text { improve accessibility }\end{array}$ \\
\hline Slide title & $\begin{array}{l}\text { Set as heading using the } \\
\text { respective HTML tag. }\end{array}$ & $\begin{array}{l}\text { Ensure that each slide has a } \\
\text { unique title. }\end{array}$ \\
\hline Text box & $\begin{array}{l}\text { Authoring toolbar creates } \\
\text { appropriate HTML tags text } \\
\text { styling, lists and hyperlinks. }\end{array}$ & $\begin{array}{l}\text { Ensure that lists and styles are } \\
\text { added using the authoring toolbar. }\end{array}$ \\
\hline Image & $\begin{array}{l}\text { Interface provided for adding } \\
\text { alt text and captions to images. }\end{array}$ & $\begin{array}{l}\text { Ensure that the alt text is } \\
\text { meaningful. Where multiple images } \\
\text { are combined to form a diagram, } \\
\text { the alt text should describe the } \\
\text { diagram appropriately. }\end{array}$ \\
\hline $\begin{array}{l}\text { Embedded } \\
\text { content }\end{array}$ & $\begin{array}{l}\text { An iframe can be accessed } \\
\text { using the keyboard, alternative } \\
\text { input devices and assistive } \\
\text { technologies. An interface is } \\
\text { provided for adding a title or } \\
\text { caption for embedded element. }\end{array}$ & $\begin{array}{l}\text { Ensure that each iframe has a title } \\
\text { and that the site provides } \\
\text { accessible controls. If the content } \\
\text { contains video or audio media, and } \\
\text { it does not have closed captions } \\
\text { and/or an audio description, then } \\
\text { a transcript and description should } \\
\text { be provided in the speaker notes or } \\
\text { attached to the slide. }\end{array}$ \\
\hline Equations & $\begin{array}{l}\text { Equations are embedded within } \\
\text { slides as MathML. }\end{array}$ & $\begin{array}{l}\text { Ensure authors avoid adding } \\
\text { equations as images. }\end{array}$ \\
\hline Tables & $\begin{array}{l}\text { Tables are created as HTML, } \\
\text { and an interface is provided for } \\
\text { setting headers and adding a } \\
\text { caption or text summary. }\end{array}$ & $\begin{array}{l}\text { Define which rows and/or columns } \\
\text { are headers and provide a caption } \\
\text { or text summary of the table. }\end{array}$ \\
\hline
\end{tabular}

\section{Approaches for Ensuring Authors Address the Accessibility Requirements of Slides}

As part of an iterative, user-centered design process, six approaches were identified for encouraging authors to improve the accessibility of their slides:

1. Require: Require authors to address accessibility issues on each slide before they save their deck. Authors would be notified of accessibility issues and the 
actions they need to undertake to make their slides accessible before they can publish their slides as OER.

2. Guidance: Assist authors to make their content accessible as they create it. Authors would be presented with guides and hints on how to make their slides more accessible as they add content.

3. Encourage: Encourage authors to check and correct accessibility issues once they have created a slide. Authors would be informed of potential accessibility issues when they attempt to save a slide and be encouraged to address the issues.

4. Rate: Encourage authors to make their slides accessible by including accessibility as a factor in rating and search results. The number of accessibility issues would be considered as a factor in rating decks and ordering search results. Decks with the least number of issues would be rated higher.

5. Crowd-source: Encourage other users to improve the accessibility of slides in an attempt to crowd-source accessibility enhancements. Users could add accessibility information to other authors' decks. This would increase their prestige on the platform as they will have contributed content. However, their changes to the deck may not be as accurate as the annotations that would have been created by the original author.

6. Automatic: Attempt to automatically fix accessibility issues. Some techniques can be used to automatically improve the accessibility of slide content, but this may result in incorrect accessibility annotations being added to slides.

Each approach presents different advantages and disadvantages to authors and users who rely on accessibility conformance. This must be balanced against the goal of encouraging authors to create OERs, with the potential to be accessible through an efficient and satisfying user experience.

In order to establish which approach would be most effective to encourage authors to create accessible slides, a survey was distributed to lecturers and teachers creating content within the SlideWiki project. Each approach was explained in the survey with an illustrate mock-up. Authors were requested to rank each approach using a five point Likert scale. In addition, the survey included a question about how much time the authors were willing to spend on accessibility issues per slide, and a free-text question were provided for additional comments. Thirteen authors responded to the survey; their results are presented in Figure 1.

The approaches "encourage" and "guidance" received the most positive responses from authors with $77 \%$ and $69 \%$ in agreement, respectively. This shows that many authors would like to be made aware of potential accessibility issues and would like information on how to address these issues. Responses for the "require" approach, which would ensure that authors addressed accessibility issues before publishing their deck, were also positive overall with $62 \%$ of respondents agreeing with this approach. Automatically correcting accessibility issues was the least popular approach with only $23 \%$ responding positively. Comments from authors indicated that they were concerned about the quality of automatic annotations and their content being altered without their approval. Similarly, 


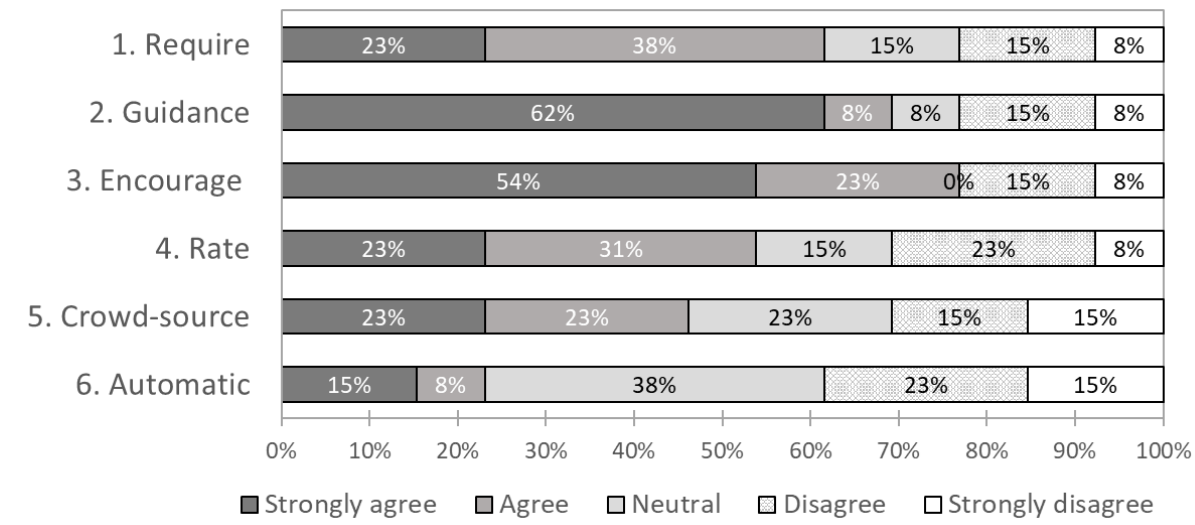

Fig. 1. Chart of the responses by slide authors to questions of whether they agreed with each proposed approach to improving the accessibility of slides.

there were mixed views on whether other users should be allowed to improve the accessibility of authors slides using the "crowd-source" approach, as concerns were raised about changes being made to authors' slides without their knowledge. Authors did not show a clear preference on whether decks should be rated on their accessibility.

Figure 2 illustrates the amount of effort that authors would be willing to spend on accessibility issues. Authors were asked to say how long they were prepared to spend on fixing accessibility issues on a slide: i) no time, ii) two minutes, iii) five minutes or iv) as long as it takes to create a slide. More than half of the respondents indicated that they felt two minutes was a reasonable time to fix accessibility issues on a slide, and $38.5 \%$ were willing to spend longer than two minutes. Only one respondent indicated they were not willing to spend any time on addressing accessibility issues.

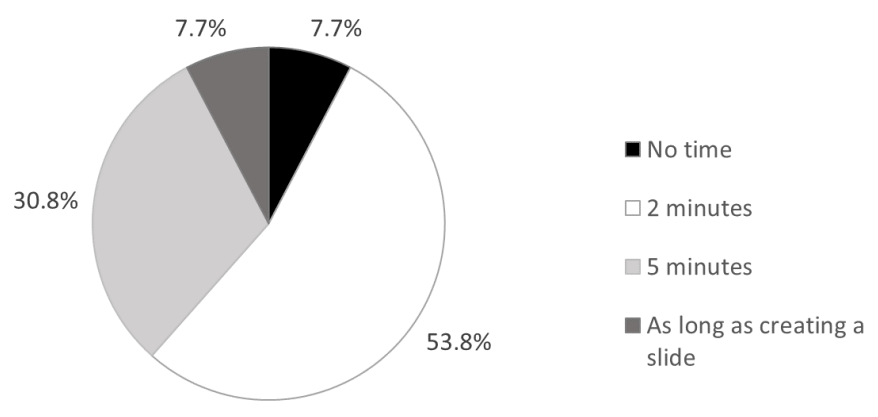

Fig. 2. Chart of responses by slide authors to the question "How long are you willing to spend on improving the accessibility of a slide?" 


\section{Discussion}

Despite slide presentations being one of the most common forms of learning material, there has been little work on investigating how these can be made accessible to all users. Accessibility guidance has tended to focus on materials that form a linear document or web page. By considering common elements in slides, six areas were identified as requiring authors input (cf. Table 1). This is a more straightforward list of requirements for authors to review than WCAG2.0 [4], which contains at least 60 success criteria.

Responses from authors indicate that there is a preference to be encouraged to improve the accessibility of slides, as long as the process is efficient and not too time consuming. However, the two approaches that are preferred by the authors ("encourage" and "guidance") would rely on their judgment and goodwill to resolve issues.

The survey was small; it was clear that quality and content ownership as well as the usability of the accessibility approach would affect how likely authors were to engage with improving the accessibility of slides. Improving accessibility cannot be separated from usability. It is important to ensure that the tools for creating accessible content are efficient and effective, as poor usability could result in a lower usage and mitigate the objective of creating more accessible content.

Of particular interest were the concerns about the quality of the automatic accessibility annotations. Authors want to be able to check and confirm any amendments to their slides before they are published. This would limit the efficiency and usability gains of automated processes. On the other hand, the use of automatically generated image descriptions is increasing, and studies of their use in social media tools have shown that blind and visually impaired users tend to accept automatic descriptions if they are aware of their possible ambiguity [10]. However, the quality and provenance of learning content is particularly important when encouraging the adoption of OERs. Therefore, having a level of ambiguity within accessibility annotations may not be appropriate in a learning context and requires further investigation.

\section{Conclusion}

Encouraging authors of OERs and OCW to consider the needs of disabled learners and to meet accessibility requirements is vital for inclusion. Despite the wide use of slide presentations in education, few studies have considered the related accessibility requirements. This paper has reviewed the elements that make up slide presentations, and has determined which of these can impact the experience of users with accessibility needs. By providing a slide editing tool for creating accessible content, there are mainly six elements commonly used within slides that require the author to undertake actions to ensure the slides are accessible. Willingness to spend time fixing accessibility issues is increased if support is offered, as long as it is an efficient process. As a result of this work, it is intended 
that the SlideWiki platform will warn authors of potential accessibility issues and provide them with guidance as they create content. The authors' concerns about automatic correction of accessibility issues should be noted and future studies are needed to consider whether the impact of providing potentially inaccurate accessibility information benefits or hinders learners.

Acknowledgments. This research has been supported by the EU project SlideWiki (grant no. 688095).

\section{References}

1. How to Make Presentations Accessible to All. Web Accessibility Initiative (WAI). https://www.w3.org/WAI/training/accessible (2012)

2. IMS Access For All. IMS Global Learning Consortium. https://www.imsglobal. org/activity/accessibility (2012)

3. Authoring Tool Accessibility Guidelines (ATAG2.0). WWW Consortium (W3C). https://www.w3.org/TR/IMPLEMENTING-ATAG20/ (2015)

4. Caldwell, B., Cooper, M., Reid, L.G., Vanderheiden, G.: Web content accessibility guidelines (WCAG2.1). https://www.w3.org/TR/WCAG20/ (2008)

5. Dattolo, A., Luccio, F.L.: A review of websites and mobile applications for people with autism spectrum disorders: Towards shared guidelines. In: International Conference on Smart Objects and Technologies for Social Good. pp. 264-273. Springer (2016)

6. Elias, M., Lohmann, S., Auer, S.: Ontology-based representation of learner profiles for accessible opencourseware systems. In: International Conference on Knowledge Engineering and the Semantic Web. pp. 279-294. Springer (2017)

7. Accessibility requirements suitable for public procurement of ICT products and services in Europe. Standard 1.1.1 (2014-02), European Telecommunications Standards Institute, France (Feb 2014)

8. Hilton III, J., Wiley, D., Stein, J., Johnson, A.: The four 'R's of openness and ALMS analysis: frameworks for open educational resources. Open Learning 25(1), 37-44 (2010)

9. James, A., Draffan, E., Wald, M.: Designing web-apps for all: How do we include those with cognitive disabilities? Studies in health technology and informatics $\mathbf{2 4 2}$, 665-668 (2017)

10. MacLeod, H., Bennett, C.L., Morris, M.R., Cutrell, E.: Understanding blind people's experiences with computer-generated captions of social media images. In: Proceedings of the 2017 CHI Conference on Human Factors in Computing Systems. pp. 5988-5999. ACM (2017)

11. Teixeira, A., Correia, C.J., Afonso, F., Cabot, A.G., López, E.G., Tortosa, S.O., Piedra, N., Canuti, L., Guzmán, J., Sol, M.Á.C.: Inclusive open educational practices: How the use and reuse of oer can support virtual higher education for all. European Journal of Open, Distance and E-learning 16(2) (2013) 